\title{
Survey on software maintenance profile and knowledge requirement in public higher learning institutions
}

\begin{abstract}
Software maintenance (SM) environment is highly complex, knowledge-driven and collaborative. Therefore, Knowledge management (KM) is critical to provide an environment for sharing and sustaining knowledge. Issues such as inadequate knowledge and lack on information sharing are still regarded as major challenges in SM. This paper presents the results of a survey on KM of SM process in selected higher learning institutions (HLIs) in Malaysia. Based on the survey, several deficiencies are identified as common to HLIs. Domain knowledge is important but these are seldom stored in KMS or other electronic means. Maintainers also spent considerable efforts collaborating with other parties to obtain information. Therefore, resolving these issues should be given high priority.
\end{abstract}

Keyword: Knowledge management (KM); Software maintenance (SM); Higher learning institutions 